\title{
Síntesis de captadores y liberadores de fertilizantes a partir de nanomateriales arcillosos'
}

\author{
Prado, B., ${ }^{*}$ Mora L., ${ }^{*}$ Millán, L. ${ }^{* *}$ y Sampieri, A. ${ }^{* * *}$
}

Resumen: El presente trabajo da alternativas para un uso más eficiente de fertilizantes primarios empleados en las prácticas agrícolas, tales como los fosfatos y los nitratos. El objetivo es proponer nanomateriales que tengan la capacidad de retener este tipo de fertilizantes y que, posteriormente, puedan liberarse de manera controlada. Para ello es necesario materiales económicos, no tóxicos y fáciles de manipular. En este sentido, las arcillas (catiónicas y aniónicas) resultan ser excelentes candidatos como materiales liberadores de fertilizantes. En este estudio, se abarcan dos puntos importantes: 1) la síntesis y la caracterización de arcillas catiónicas y aniónicas susceptibles de retener fosfatos y nitratos $y, 2$ ) la capacidad y eficiencia que tienen estos materiales para liberar estos fertilizantes en solución acuosa.

\section{INTRODUCCIÓN}

El nitrógeno y el fósforo son elementos esenciales para el desarrollo de los cultivos. Sin embargo, algunos de ellos como el nitrato y ciertas formas de fosfatos son, además, contaminantes de cuerpos de agua superficiales y profundos. La forma más común para suministrar estos elementos minerales a los cultivos es el uso de enmiendas orgánicas o de fertilizantes. En la mayoría de los casos se da un uso inadecuado o excesivo de fertilizantes en las prácticas agrícolas, lo que propicia la contaminación de los cuerpos de agua. Con el objeto de definir un mejor uso de los fertilizantes se ha propuesto el empleo de fertilizantes de lenta liberación, los cuales presentan un gran potencial tanto en la agricultura como en la reforestación (Rose et al., 2004; Wu y Liu, 2007; Wu et al., 2008). Sin embargo, este tipo de fertilizantes todavía presenta un alto costo de producción y, en ciertos casos, puede ocurrir una liberación descontrolada o ineficiente del mismo. Una de las formas de evitar tales inconvenientes, sin dejar de lado la función fertilizadora, es el uso de materiales nanoestructurados capaces de liberar nutrientes de forma gradual. Esto no sólo reduciría la contaminación de suelos y agua, sino que también significaría un ahorro económico altamente considerable. Este trabajo se enfoca en la preparación de arcillas aniónicas, también conocidas como hidrotalcitas, y de arcillas catiónicas que tengan la capacidad de retener y de liberar gradualmente fertilizantes (nitrato y fosfato) en solución acuosa.

1 Agradecimientos. La investigación fue realizada con financiamiento de los proyectos PAPIIT IN116310, CONACYT 99964 y SEP-PROMEP/103.5/09/4194 .

* Instituto de Geología, Universidad Nacional Autónoma de México, Cd. Universitaria, México, D.F., 04510, México.

** Instituto Tecnológico de Estudios Superiores de Chimalhuacán, Ingeniería Química, Chimalhuacán, 56230, Estado de México.

*** Benemérita Universidad de Puebla, Facultad de Ingeniería Química, Av. San Claudio, Ciudad Universitaria, Puebla, 72527, México. E-mail: asamcr@yahoo.com 
Las arcillas son minerales muy abundantes en la naturaleza, las cuales han sido utilizadas por el hombre desde hace más de veinticinco mil años (Fetter y Castillo, 2005). Nuestros antiguos pobladores de Mesoamérica ya las empleaban en la preparación de vasijas y casas de adobe. Actualmente, la aplicación de las arcillas es muy importante debido a su gran abundancia en la naturaleza. Sus materiales son tan versátiles pues presentan gran estabilidad térmica e inocuidad, además de tener capacidades adsorbentes y absorbentes. Dichas propiedades permiten la aplicación de las arcillas en todos los campos de la ciencia y la tecnología, como la medicina, la biología, la química de materiales y por supuesto, la agronomía (Carretero, 2002; Choy et al., 2007; Dasgupta y Torok, 2008).

En general, la arcillas se pueden clasificar en dos grandes grupos en arcillas catiónicas y arcillas aniónicas.

\section{Arcillas catiónicas}

Este tipo de arcillas presentan una estructura laminar conformada por silicatos de aluminio y magnesio hidratados, es decir, $\mathrm{Si}, \mathrm{O}, \mathrm{Al}, \mathrm{Mg} \mathrm{y}_{2} \mathrm{O}$, principalmente. Aunque por lo general presentan otros metales en menor proporción, como el hierro. Debido a su porosidad interna y a su carga electrostática, las arcillas permiten la sorción (adsorción y absorción) de cationes simples, como, por ejemplo, potasio, magnesio y amonio, los cuales pueden liberarse o intercambiarse en medio ácido (Kloprogge et al., 1999; Zhang et al., 2010). Las esmectitas son las arcillas que presentan propiedades interesantes para su empleo como adsorbentes. La montmorillonita es la arcilla catiónica tipo esmectita más representativa (figura 1). Su estructura consiste de una capa octaédrica de hidragilita-brucita $\left[\mathrm{Al}(\mathrm{OH})_{3}-\mathrm{Mg}(\mathrm{OH})_{2}\right]$ incluida entre dos capas

FIGURA 1. Estructura de una arcilla catiónica

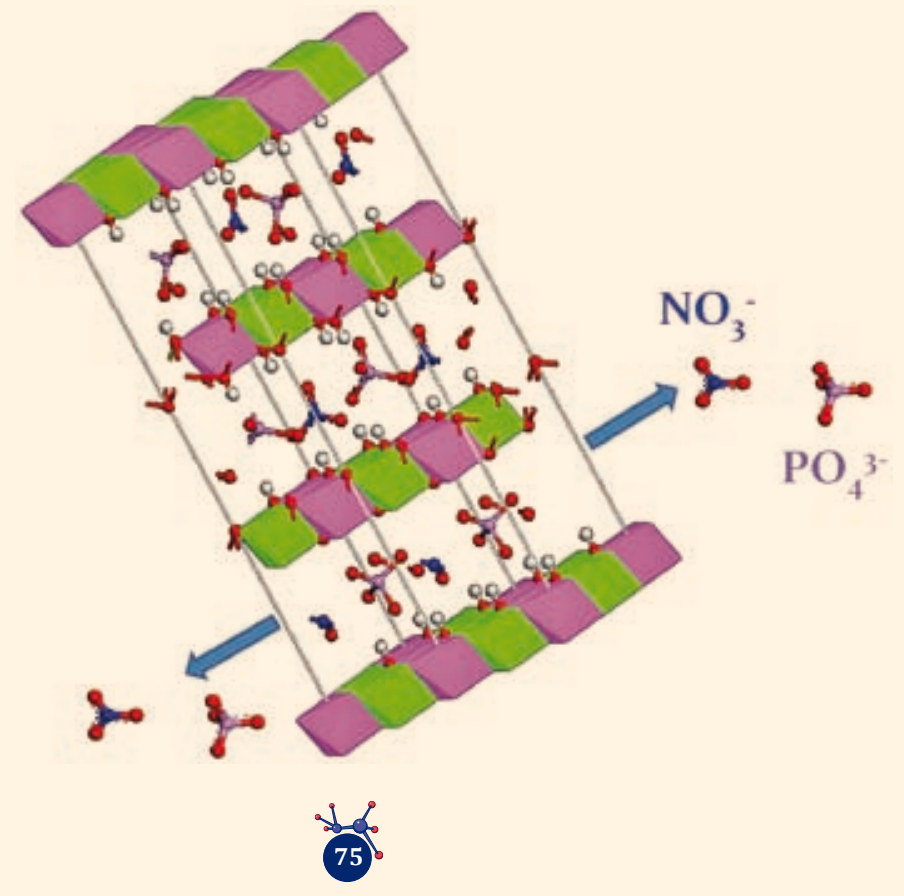


tetraédricas de silicio. Las capas octaédricas presentan un exceso de cargas negativas originadas por la sustitución natural de algunos átomos de $\mathrm{Al}^{3+}$ por átomos de $\mathrm{Mg}^{2+}$. Estas cargas se compensan por cationes localizados en la superficie interlaminar, generalmente, $\mathrm{Na}^{+}, \mathrm{K}^{+}, \mathrm{Ca}^{2+} \mathrm{y} / \mathrm{o} \mathrm{NH}_{4}{ }^{+}$, denominados cationes intercambiables o cationes de compensación de carga. La principal característica de la montmorillonita es que en su espacio interlaminar puede penetrar tanto el agua como moléculas orgánicas e inorgánicas, causando una expansión entre las láminas. Estas arcillas tienen una capacidad de intercambio catiónico (CIC) de 60-120 miliequivalentes por 100 gramos de material.

\section{Arcillas aniónicas}

La hidrotalcita es un mineral cuya fórmula química estructural es $\left[\mathrm{Mg}_{6} \mathrm{Al}_{2}(\mathrm{OH})_{16}\right]$ $\left(\mathrm{CO}_{3}\right) \bullet 4 \mathrm{H}_{2} \mathrm{O}$. Este mineral pertenece al grupo de las arcillas aniónicas. La estructura de la hidrotalcita es similar a la de la brucita, $\mathrm{Mg}(\mathrm{OH})_{2}$, en la cual el magnesio se encuentra coordinado octaédricamente a seis grupos hidroxilo (OH').(Liy Duan, 2006; Lima, 2004) Estos octaedros forman láminas bidimensionales (figura 2) Cuando algunos cationes $\mathrm{Mg}^{2+}$ se reemplazan por $\mathrm{Al}^{3+}$, se forma la hidrotalcita y el arreglo laminar adquiere cargas excedentes positivas. Para compensar esta carga se requiere de un anión; en el caso de la hidrotalcita, son los carbonatos $\mathrm{CO}_{3}{ }^{2-}$ que se localizan en región interlaminar, junto con moléculas de agua. Las hidrotalcitas o arcillas aniónicas (figura 2) pueden sintetizarse fácilmente en el laboratorio. La variedad de familias de compuestos tipo hidrotalcita (HT) que se puede preparar es enorme y la fórmula general que los representa es: $\mathrm{M}^{2+}{ }_{1-\mathrm{x}} \mathrm{M}^{3+}{ }_{\mathrm{x}}(\mathrm{OH})_{2} \mathrm{~A}^{\mathrm{n}-}{ }_{\mathrm{x} / \mathrm{n}} \bullet \mathrm{yH}_{2} \mathrm{O}$ en donde $\mathrm{M}^{2+} \mathrm{y} \mathrm{M}^{3+}$ son cationes metálicos divalentes $\mathrm{y}$ trivalentes, respectivamente, mientras que $\mathrm{A}^{\mathrm{n}-\mathrm{-}}$ representa un anión de carga $\mathrm{n}$. Este

FIGURA 2. Estructura de una arcilla aniónica

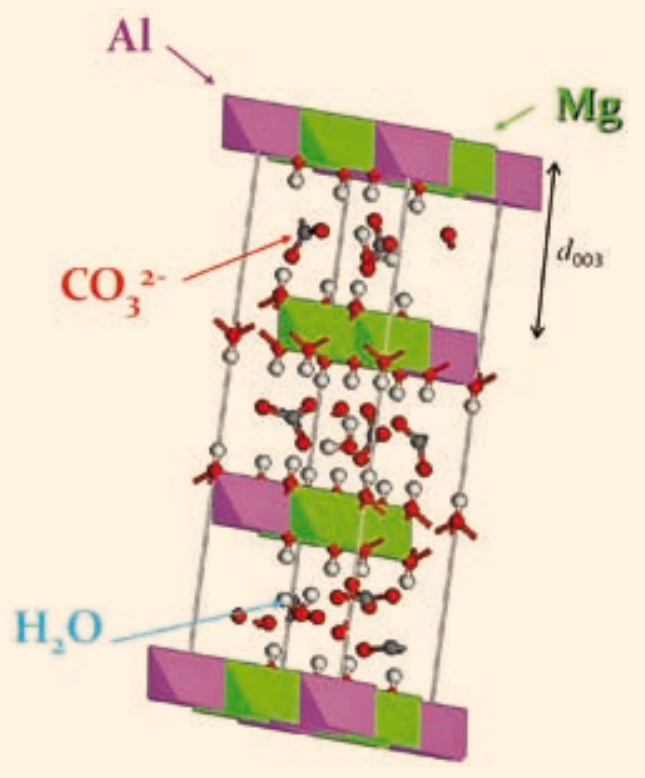


anión interlaminar puede ser intercambiado por muchos otros en medio acuoso u orgánico (Li y Duan, 2006). El valor de x representa la fracción de cationes del metal trivalente, usualmente esta fracción se encuentra entre 0.20 y 0.35 .

En nuestro caso, se preparan arcillas aniónicas de magnesio y de aluminio con nitratos o con fosfatos como aniones compensadores de carga. Además, se empleó también una montmorillonita para retener los cationes de amonio, procedente del hidróxido de amonio $\mathrm{NH}_{4} \mathrm{OH}$, que se emplea durante la síntesis de las hidrotalcitas. La capacidad de liberación (desorción) de nitratos y de fosfatos, contenidos en estos materiales, se estudia en solución acuosa. Para ello, se emplean diferentes tiempos de contacto y se evalúa la capacidad o tasa de desorción en función del tiempo de liberación. Los aniones liberados (nitratos y fosfatos) se determinaron por cromatografía líquida de alta resolución (high performance liquid chromatography, HPLC). Los resultados muestran que los materiales evaluados liberan nitrato y fosfato en forma gradual.

\section{SÍNTESIS Y CARACTERIZACIÓN DE LOS MATERIALES}

\section{LIBERADORES DE NUTRIENTES}

Un buen diseño de materiales arcillosos que contengan nutrientes capaces de liberarse lentamente en el suelo es fundamental. Además, su elaboración debe de ser factible en términos económicos. Por tal motivo, se decidió estudiar la capacidad que tienen las arcillas catiónicas y aniónicas de retener nutrientes en forma iónica, como nitrato $\left(\mathrm{NO}_{3}{ }^{-}\right)$, fosfatos $\left(\mathrm{PO}_{4}{ }^{3-}\right)$ o amonio $\left(\mathrm{NH}_{4}^{+}\right)$. Además, la elaboración de estos "materiales nutrientes" es relativamente sencilla y económicamente viable para su uso en la agricultura.

La síntesis de la arcilla aniónica con nitratos (Hidrotalcita- $\mathrm{NO}_{3}^{-}$) se preparó siguiendo el método de coprecipitación propuesto por Olanrewaju y cols. (Olanrewaju et al., 2000), el cual consiste en precipitar, de manera simultánea, una solución acuosa ( 2 molar) que contenga sales de nitratos de aluminio y de magnesio con otra solución de hidróxido de amonio ( 2 molar). Este último es el agente precipitante. Con el fin de asegurar la mayor concentración posible de nitratos interlaminares, la relación molar $x=\mathrm{Al}^{3+} / \mathrm{Al}^{3+}+\mathrm{Mg}^{2+}$ debe de ser de 0.33 . Esto también contribuye a una estructura laminar estable de la arcilla aniónica. La adición de las dos soluciones (sales e hidróxido) se lleva a cabo lenta y simultáneamente a un recipiente en agitación constante, manteniendo un pH de 9 en todo el proceso. Durante este procedimiento, las sales de $\mathrm{Mg}$ y de $\mathrm{Al}$ precipitan, se caracterizan porque presentan un color blanco lechoso. Una vez adicionada la totalidad de ambas soluciones se deja agitar durante 24 horas aproximadamente. Con este tiempo se permite que los hidróxidos de Mg y Al precipiten, y se ordenen para formar la estructura laminar característica de una hidrotalcita (proceso conocido como condensación estructural), en cuya región interlaminar se disponen los aniones compensadores de cargas, es decir, los nitratos $\left(\mathrm{NO}_{3}{ }^{-}\right)$. Después de la agitación, el sólido de color blanco se separa por centrifugación del exceso de solución, se lava para eliminar el remanente de nitratos, y se seca a $60^{\circ} \mathrm{C}$. ¡El material está listo! La ventaja de este método es que los $\mathrm{NO}_{3}{ }^{-}$contenidos en las sales de $\mathrm{Al}$ y de Mg son ahora los aniones compensadores de cargas que posteriormente serán liberados como fertilizantes.

La síntesis de la arcilla aniónica con fosfatos se preparó de manera similar al método anterior, con la diferencia de que una tercera solución de acuosa de fosfato diácido 
de amonio, $0.03 \mathrm{M}\left[\left(\mathrm{NH}_{4}\right) \mathrm{H}_{2} \mathrm{PO}_{4}\right]$ se agregó simultáneamente junto con las soluciones de las sales de $\mathrm{Mg}$ y de $\mathrm{Al}$, así como el agente precipitante $\left(\mathrm{NH}_{4} \mathrm{OH}\right)$. Es decir, ahora esperamos obtener una hidrotalcita con iones fosfatos en su interior, $\mathrm{PO}_{4}{ }^{3-}$.

Debido a que el ion amonio, $\mathrm{NH}_{4}{ }^{+}$(presente en el hidróxido de amonio, $\mathrm{NH}_{4} \mathrm{OH}$ ), también puede ser una fuente de nutriente para las plantas, se puede recuperar mediante el uso de una arcilla catiónica u otro material intercambiador de cationes, como las zeolitas. Ya sea después o durante la preparación de las hidrotalcitas. En nuestro caso, se empleó una bentonita comercial, una montmorillonita, que al ponerse en contacto con las soluciones acuosas que contiene iones $\mathrm{NH}_{4}{ }^{+}$, éstos se intercambian por los cationes de potasio y calcio presentes en la arcilla. Sin embargo, la capacidad de retención de los iones $\mathrm{NH}_{4}{ }^{+}$dependerá en gran medida de la capacidad de intercambio catiónico de la arcilla, que es de aproximadamente $100 \mathrm{meq} / 100 \mathrm{~g}$. Por lo tanto, se decidió sintetizar a $\mathrm{pH}=9$, una hidrotalcita o arcilla aniónica de $\mathrm{Mg}-\mathrm{Al}$ con nitratos, tal y como se describió anteriormente. Pero ahora, la mezcla de sales metálicas y del agente precipitante, no están solas. En efecto, una mezcla de una arcilla catiónica y agua desionizada se utilizó durante la síntesis. La relación molar de Bentonta-Hidrotalcita- $\mathrm{NO}_{3}{ }^{-}$fue de 50:50. Con ello se pretende, primero, recuperar una parte de los iones $\mathrm{NH}_{4}^{+}$presentes en el hidróxido empleado para llevar a cabo la coprecipitación de las sales de $\mathrm{Mg}$ y de $\mathrm{Al}$, y segundo, los iones nitratos presentes en la arcilla catiónica.

Gracias a estos tres sencillos métodos de preparación se lograron obtener materiales relativamente económicos que contienen nutrientes esenciales para las plantas, específicamente, iones que contienen nitrógeno y fósforo. Sin embargo, la composición no fue la misma en ninguno de los tres casos. Para una mejor comprensión de esto, las capacidades de retención de los nutrientes para cada uno de los materiales se resumen en la tabla 1 preparados. La arcilla aniónica con nitratos, Hidrotalcita- $\mathrm{NO}_{3}{ }^{-}$presenta una buena correlación de la concentración esperada de nitratos (concentración teórica) y la concentración real $(0.17 \mathrm{~g} / \mathrm{g}$ ). Para el caso de la arcilla aniónica con fosfatos, la concentración teórica corresponde únicamente a la presen-

TABLA 1. Capacidad de retención de los nutrientes

\begin{tabular}{|c|c|c|c|c|c|}
\hline \multirow{2}{*}{$\begin{array}{l}\text { Muestra de } \\
\text { arcilla }\end{array}$} & \multirow{2}{*}{ Relación molar } & \multirow{2}{*}{ Nutriente } & \multicolumn{3}{|c|}{$\begin{array}{c}\text { Concentración de nutriente } \\
\qquad(\mathrm{g} / \mathrm{g})^{*}\end{array}$} \\
\hline & & & (teórica) & $\begin{array}{c}(\text { real })^{* *} \\
\mathrm{NO}_{3}^{-}\end{array}$ & $\begin{array}{c}(\text { real) } \\
\mathrm{PO}_{4}{ }^{*-}\end{array}$ \\
\hline $\begin{array}{l}\text { Hidrotalcita- } \\
\mathrm{NO}_{3}^{-}\end{array}$ & $\frac{\mathrm{Al}^{3+}}{\mathrm{Al}^{3+}+\mathrm{Mg}^{2+}}=0.33$ & $\mathrm{NO}_{3}^{-}$ & 0.23 & 0.17 & - \\
\hline $\begin{array}{l}\text { Hidrotalcita- } \\
\mathrm{PO}_{4}^{3-}\end{array}$ & $\frac{\mathrm{Al}^{3+}}{\mathrm{Al}^{3+}+\mathrm{Mg}^{2+}}=0.33$ & $\mathrm{PO}_{4}^{3-}$ & 0.22 & 0.18 & 0.22 \\
\hline $\begin{array}{c}\text { Bentonita- } \\
\text { Hidrotalcita } \\
\mathrm{NO}_{3}^{-}\end{array}$ & $\frac{\text { Bentonita }}{\text { Bentonita-Hidrotalcita }}=0.5$ & $\mathrm{NH}_{4}^{+}+\mathrm{NO}_{3}^{-}$ & 0.16 & 0.044 & - \\
\hline
\end{tabular}

* gramo de nutriente por gramo de material

** determinado por conductividad térmica en un analizador elemental CNHS Perkin Elmer 2400. 
cia de estos aniones. Sin embargo, la concentración real demuestra que existen tanto iones $\mathrm{NO}_{3}{ }^{-}(0.18 \mathrm{~g} / \mathrm{g})$ como $\mathrm{PO}_{4}{ }^{3-}(0.22 \mathrm{~g} / \mathrm{g})$. Este último resultado es algo ambiguo, en el sentido de que una parte de estos aniones pueden localizarse entre las láminas y otra parte en la superficie del material: ¡hay muchos más nutrientes de lo esperado! En efecto, la suma de los dos resulta ser demasiado grande para encontrase en su totalidad entre las láminas de la arcilla. La concentración teórica y la real, de la mezcla Bentonta-Hidrotalcita- $\mathrm{NO}_{3}{ }^{-}$, corresponden a la suma de los dos nutrientes nitrogenados que estarían presentes dentro del material, es decir, $\mathrm{NH}_{4}{ }^{+} \mathrm{yNO}_{3}{ }^{-}$. Desafortundamente, la mezcla de una arcilla catiónica y una arcilla aniónica, no se traduce en un material con un buen contenido de iones nitrogenados. Más adelante se explica dicho comportamiento.

La arcilla aniónica con nitratos, Hiudrotalcita- $\mathrm{NO}_{3}^{-}$entre sus láminas presenta una estructura laminar relativamente homogénea que se demuestra por la presencia del pico de difracción (003). Este pico es intenso y bien definido y, también, es representativo de la distancia interlaminar de la hidrotalcita con nitratos (figura 3, Hidrotalcita- $\mathrm{NO}_{3}^{-}$), cuyo valor es $d_{003}=8.9 \AA$ (Amstrong, $1 \AA=1 \times 10^{-10} \mathrm{~m}$ ). Ahora bien, la hidrotalcita con fosfatos, $\mathrm{PO}_{4}{ }^{3-}$, presenta una estructura menos ordenada que la anterior, el pico (003), es mucho más ancho y menos intenso que en el caso de los nitratos. Sin embargo, la distancia interlaminar es de $9.2 \AA$, ligeramente mayor a la hidrotalcita únicamente con nitratos. Ello indica que los fosfatos están presentes en la región interlaminar. Aunque, de acuerdo con los resultados de la tabla 1, no sólo existen nitratos sino también fosfatos. Por lo tanto, en la región interlaminar existe una mezcla de fosfatos y nitratos contenidos en la parte interlamianar de la arcilla (los fosfatos tienen un radio iónico mayor que los nitratos). Recordemos que durante la síntesis

FIGURA 3. Difractogramas de rayos $X$ de las arcillas con nutrientes entres sus láminas, la arcilla catiónica, una bentonita comercial, se muestra como referencia.

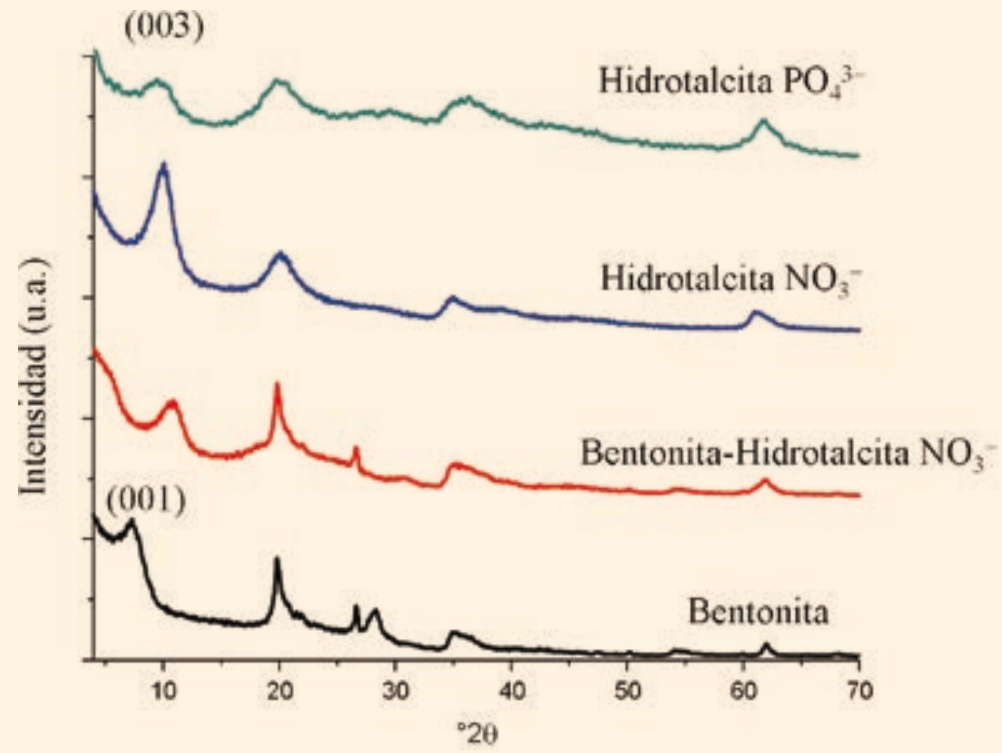


de este material se emplean sales de nitratos de $\mathrm{Mg}$ y Al, además de una solución de $\left(\mathrm{NH}_{4}\right) \mathrm{H}_{2} \mathrm{PO}_{4}$ por lo que no resulta extraño encontrar las dos especies en el mismo material. Ese resultado podría ser benéfico si se quieren preparar materiales con más de un nutriente. Para el caso de la mezcla 50:50 de bentonta-hidrotalcita- $\mathrm{NO}_{3}{ }^{-}$se observa que el pico (003) de la arcilla aniónica se mantiene, pero disminuye la distancia interlaminar, $\left(d_{003}=7.8 \AA\right)$. Sin embargo, el pico (001) característico de la bentonita comercial, no aparece lo que indica que su estructura colapsó durante la preparación de la hidrotalcita con nitratos. Ello puede deberse a que el pH de síntesis fue demasiado alto $(\mathrm{pH}=9)$. En consecuencia, la mezcla de arcillas provoca una baja concentración de nitrógeno (tabla 1), el cual debe estar principalmente en forma de $\mathrm{NO}_{3}{ }^{-}$, el resto de los aniones compensadores de carga son, casi con certeza, carbonatos $\mathrm{CO}_{3}{ }^{2-}$. En efecto, los carbonatos tienen una mayor afinidad a intercalarse en una arcilla aniónica que cualquier otro anión y propician una distancia interlaminar, $d_{003}$ de $7.7 \AA$, muy cercana a la determinada en nuestro caso que fue de $7.9 \AA$. Es decir, el $\mathrm{CO}_{3}{ }^{2-}$ tiene un radio iónico menor que el de $\mathrm{NO}_{3}{ }^{-}$. Como vemos, no siempre se logra lo que se pretende, la recuperación del amonio mediante este método todavía merece ser estudiada a profundidad. Se sabe que la bentonita (sin hidrotalcita) en contacto con una solución de iones $\mathrm{NH}_{4}^{+}$(a partir de $\mathrm{NH}_{4} \mathrm{OH}$ ) sí son retenidos en la parte interlaminar de la misma. Sin embargo, en presencia de sales de $\mathrm{Mg}$ y Al, y de hidróxido de amonio, sólo conduce a un colapso de la estructura laminar de la bentonita. A pesar de ello, se estudió para cada una de los tres sólidos su capacidad de liberación de nutrientes en agua.

\section{TASA DE LIBERACIÓN DE NUTRIENTES}

La evaluación de la tasa de liberación de nutrientes de los materiales sintetizados se realizó por lotes (sistema batch). Se evaluaron los materiales sintetizados y mezclas de ellos, incluyendo a la bentonita comercial como material de referencia. El experimento fue diseñado para 1) conocer el tiempo de equilibrio del sistema (cuando la concentración en solución y en el sólido no cambia con el tiempo); 2) conocer el rendimiento (porcentaje de nutriente liberado del total contenido en el material sintetizado) y, 3) evaluar la tasa de liberación (mligramo de nutriente liberado por gramo de material por hora); y evaluar el número de equilibrios o "riegos" que soporta cada muestra.

El procedimiento incluyó la agitación del material evaluado en una solución que contenía agua destilada o una solución acusa de cloruro de calcio $\left(\mathrm{CaCl}_{2}, 8 \mathrm{mM}\right.$, milimolar), a una relación 1:100 material:solución. Para la agitación constante de 120 rpm con ayuda de un agitador de tipo orbital. Durante todo el procedimiento se midió el pH observando que se mantuvo estable en todas las etapas (alrededor de 7). Se evaluaron diferentes tiempos de contacto, a cada tiempo la mezcla fue centrifugada a 2,500 rpm durante 25 min y el sobrenadante fue filtrado con ayuda de un filtro con una porosidad de $0.22 \mu \mathrm{m}$ (micrómetros). La determinación de nitratos y fosfatos liberados se hizo por cromatografía líquida de alta resolución (HPLC), en un equipo de marca Waters. Siguiendo el procedimiento señalado, se evaluó la cinética de desorción (liberación), la capacidad de liberación de los nutrientes (nitrato y fosfato) a partir de los materiales, a tiempo variable de agitación. Asimismo, se estudio la liberación de los nutrientes a tiempo constante.

En un primer tiempo se evaluó la cinética de desorción, en solución acuosa, que permitiera definir el tiempo en que el sistema alcanza el equilibrio (tiempo en que la 
FIGURA 4. Cinética de liberación de nitrato a partir de los materiales sintetizados

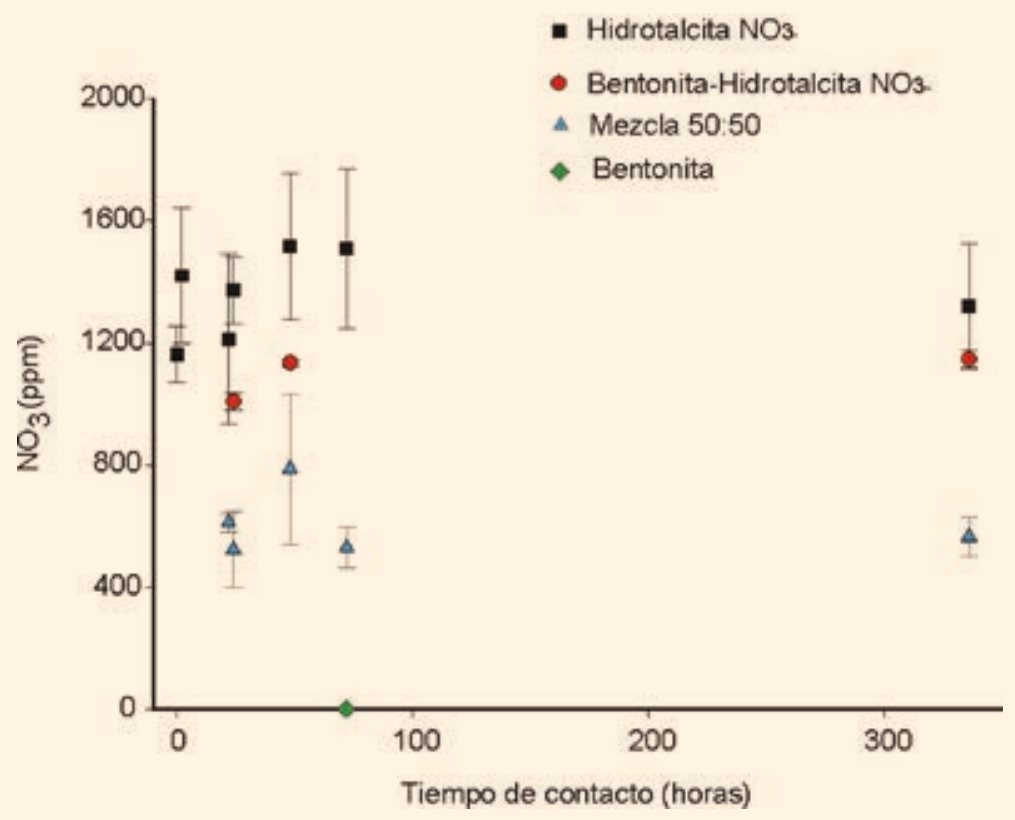

concentración de nutrientes liberada no cambia con la determinada en la solución). Se evaluaron 8 tiempos de contacto entre 0 y 1,250 horas (52 días). El equilibrio de la desorción de $\mathrm{NO}_{3}{ }^{-}$en agua se obtiene en las primeras 150 h (6.25 días). La concentración máxima liberada es de $300 \mathrm{ppm}$ de $\mathrm{NO}_{3}$. La figura 4 muestra la cinética de desorción de nitratos a partir de la arcilla aniónica, Hidrotalcita- $\mathrm{NO}_{3}^{-}$, y la mezcla Bentonita-Hidrotalcita- $\mathrm{NO}_{3}^{-}$. La figura 5 muestra en detalle la liberación de nitratos para la arcilla aniónica. Es interesante observar que la liberación de nitratos, en esta muestra, permanece constante durante los primeros seis días (144 h). La arcilla aniónica, Hidrotalcita- $\mathrm{NO}_{3}{ }^{-}$resulta ser el material que libera una cantidad de nitrato hasta 1,400 ppm de $\mathrm{NO}_{3}$. En los materiales evaluados la concentración máxima liberada correspondió al $47.80 \%$ en el material Hidrotalcita- $\mathrm{NO}_{3}^{-}$.

En el análisis de la liberación de fosfatos a partir de los materiales sintetizados, se observó que al entrar en contacto con la solución de $\mathrm{CaCl}_{2}$, la hidrotalcita fosfatada, Hidrotalcita- Hidrotalcita- $\mathrm{PO}_{4}{ }^{3}$ - libera casi instantáneamente una concentración de fosfato de $90 \mathrm{ppm}$, la cual representa al $2.70 \%$ del contenido de fósforo total del material (figura 6). Después de la tercera hora de contacto, la concentración liberada osciló entre 20 y $30 \%$ y se mantuvo constante hasta las 80 horas experimentales.

En las gráficas mostradas hasta ahora se observa el alcance de la desorción de los nutrientes en un solo tiempo de contacto entre el material y la solución de $\mathrm{CaCl}_{2}$. Tal resultado se interpreta como la cantidad de nutrientes $\left(\mathrm{NO}_{3}^{-} \mathrm{y}_{4}{ }^{3-}\right)$ que serían liberados en un sólo riego del suelo cultivado, al cual se le han dosificado estos materiales sintetizados. La tasa de liberación de $\mathrm{NO}_{3}{ }_{3}$, de los materiales sintetizados se evaluó también a varios tiempos de contacto de forma consecutiva, a cada tiempo el sobrena- 
FIGURA 5. Cinética de liberación de nitrato a partir de la muestra Bentonita-Hidrotalcita $\mathrm{NO}_{3}{ }^{-}$

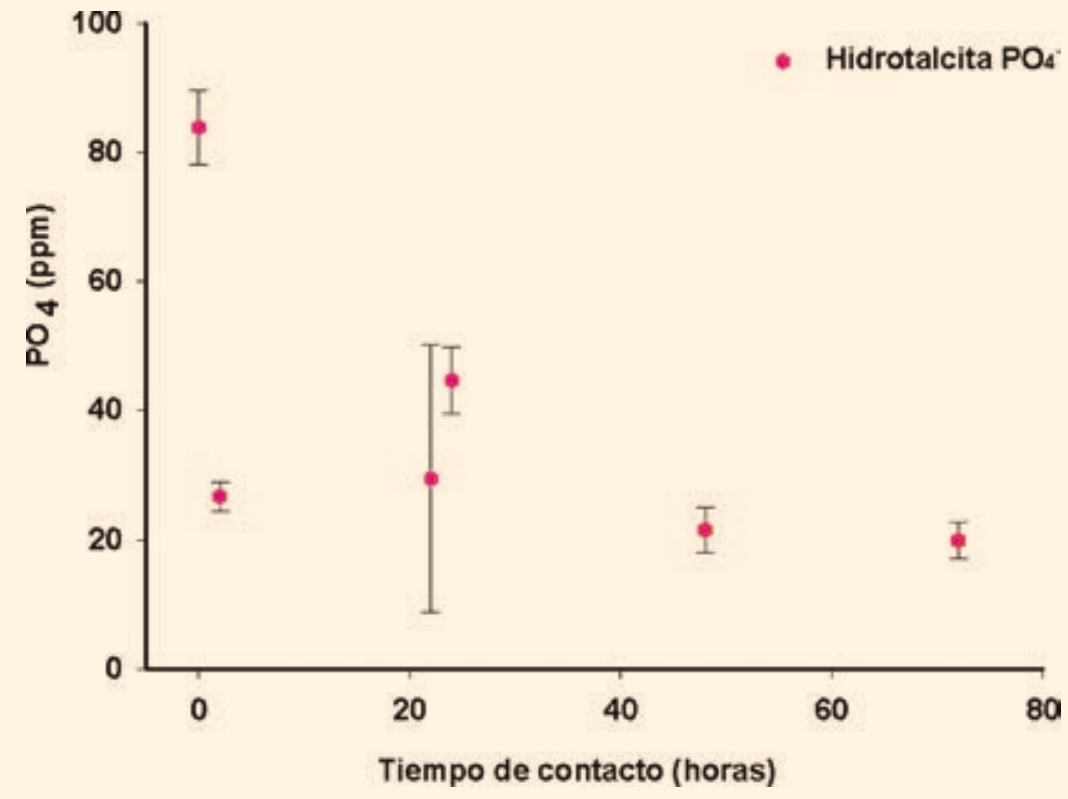

FIGURA 6. Concentración de fosfato liberado de la muestra Hidrotalcita- $\mathrm{PO}_{4}{ }^{3-}$

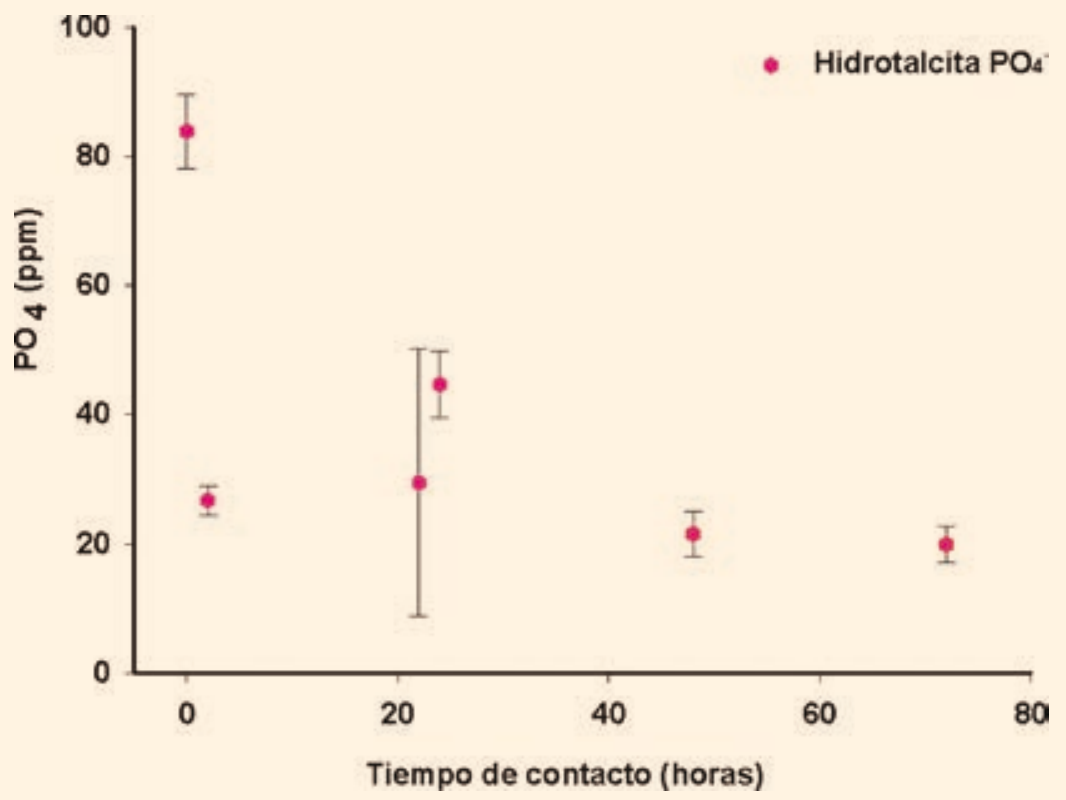


dante (solución en equilibrio) era remplazado por solución de $\mathrm{CaCl}_{2}(8 \mathrm{mM})$. En este caso, el interés es simular el comportamiento de los materiales bajo el efecto de diferentes riegos para definir la cantidad de nutrientes puestos en solución, que equivalen a la fracción disponible para las plantas. Los resultados observados bajo estas condiciones se pueden resumir de la siguiente manera: la hidrotalcita fosfatada, Hidrotalcita- $\mathrm{PO}_{4}{ }^{3-}$, libera todo el nutriente en las primeras 24 horas; la mezcla 50:50 libera gran cantidad de $\mathrm{NO}_{3}{ }^{-}$en las primeras 24 horas (>1500 ppm) y mantiene su almacén hasta el siguiente evento de riego ( $>400 \mathrm{ppm})$; la muestra Hidrotalcita- $\mathrm{NO}_{3}{ }^{-}$libera menor cantidad de nitratos ( 300 ppm) en 2 horas, sin embargo, mantiene su almacén hasta el último riego. Lo obtenido indica que existen diferencias entre los materiales sintetizados vis a vis y la velocidad de liberación de los nutrientes. Los materiales sintetizados en fosfatos se agotan en un tiempo de contacto, mientras que en los materiales sintetizados con nitrato se observa una liberación más lenta y controlada, sugiriendo una mayor eficiencia al utilizarlos como materiales fertilizantes.

\section{CONCLUSIONES Y PERSPECTIVAS}

1. Se presenta un método sencillo y económico de síntesis de materiales arcillosos nanoestructurados que pueden liberar fertilizantes de manera controlada.

2. Los niveles de concentración de los nutrientes de los materiales evaluados están alrededor de $1400 \mathrm{ppm} \mathrm{de} \mathrm{NO}_{3}{ }^{-}$equivalentes a $40 \mathrm{mgN} / \mathrm{g}$ mat. (miligramos de nitrógeno por gramo de material) y de 90 ppm $\mathrm{PO}_{4}{ }^{3-}(2.8 \mathrm{mg} \mathrm{P} / \mathrm{g}$ mat). Por ejemplo, si se desea fertilizar un cultivo que requiere $0.3 \mathrm{gN} / \mathrm{m}^{2}$, sólo sería necesario aplicar $7.5 \mathrm{~g}$ del material preparado, es decir, para un área de $100 \mathrm{~m}^{2}$ de cultivo sólo sería necesario 0.75 kg de arcilla aniónica. Además, el contenido de nitrógeno y fósforo puede ser predeterminado en la síntesis de los materiales, es decir, se pueden obtener materiales con las concentraciones deseadas para un determinado tipo de cultivo.

3. La liberación de la mayor masa de nitrógeno y fosforo contenido en los materiales sintetizados ocurre de forma inmediata al entrar en contacto con la solución, tal resultado es positivo al considerarse que los nutrientes deben ser puestos a disposición de la planta de forma inmediata con el riego.

4. La siguiente etapa del trabajo será evaluar la liberación de nitrógeno y fósforo en condiciones experimentales más cercanas a lo que ocurre en una práctica agrícola al fertilizar un suelo. Se sugieren experimentos en maceta bajo condiciones de invernadero, de tal manera que sea posible definir la cantidad de nutrimentos liberados a partir de los materiales sintetizados, la cantidad lixiviada y la cantidad tomada por la planta.

\section{RefERENCIAS}

Carretero, M. I. (2002). “Clay minerals and their beneficial effects upon human health. A review". Applied Clay Science, vol. 21, núm. 3-4: 155.

Choy, J.-H., Choi, S.-J., Oh, J.-M., y Park, T. (2007). “Clay minerals and layered double hydroxides for novel biological applications". Applied Clay Science, vol. 36, núm. 1-3: 122. 
Dasgupta, S., y Torok, B. (2008). "Application of clay catalysts in organic synthesis. A review". Organic Preparations and Procedures International, vol. 40, núm. 1: 1.

Fetter, G., y Castillo, P. S. (2005). “Catalizadores tipo arcillas pilareadas”. Materiales Avanzados, vol. 4: 19.

Kloprogge, J. T., Komarneni, S., y Amonette, J. E. (1999). "Synthesis of smectite clay minerals: A critical review”. Clays and Clay Minerals, vol. 47, núm. 5: 529-554.

Li, F., y Duan, X. (2006). "Layered Double Hydroxides: Applications of Layered Double Hydroxides". Struct. Bond, 193.

Lima, E. (2004). "Los hidróxidos dobles laminares, materiales con memoria”. Materiales Avanzados, vol. 2: 31.

Olanrewaju, J., Newalkar, B. L., Mancino, C., y Komarneni, S. (2000). “Simplified synthesis of nitrate form of layered double hydroxide". Materials Letters, vol. 45, núm. 6: 307.

Rose, R., Haase, D. L., y Arellano, E. (2004). "Fertilizantes de entrega controlada: potencial para mejorar la productividad de la reforestación”. BOSQUE, vol. 25, núm. 2, 89.

Wu, L., and Liu, M. Z. (2007). "Slow-release potassium silicate fertilizer with the function of superabsorbent and water retention". Industrial \& Engineering Chemistry Research, vol. 46, núm. 20: 6494.

Wu, L., Liu, M. Z., y Liang, R. (2008). "Preparation and properties of a double-coated slowrelease NPK compound fertilizer with superabsorbent and water-retention". Bioresource Technology, vol. 99, núm. 3: 547.

Zhang, D., Zhou, C. H., Lin, C. X., Tong, D. S., y Yu, W. H. (2010). “Synthesis of clay minerals". Applied Clay Science, vol. 50, núm. 1: 1. 